\title{
Comparison of different methods for imputing genome-wide marker genotypes in Swedish and Finnish Red Cattle
}

\author{
P. Ma, ${ }^{*}{ }^{1}$ R. F. Brøndum, ${ }^{*}$ Q. Zhang,, M. S. Lund, ${ }^{*}$ and G. Su${ }^{* 1}$ \\ *Centre for Quantitative Genetics and Genomics, Department of Molecular Biology and Genetics, Aarhus University, DK 8830 Tjele, Denmark \\ tKey Laboratory of Animal Genetics and Breeding of the Ministry of Agriculture, Department of Animal Genetics and Breeding, \\ College of Animal Science and Technology, China Agricultural University, China 100193
}

\section{ABSTRACT}

This study investigated the imputation accuracy of different methods, considering both the minor allele frequency and relatedness between individuals in the reference and test data sets. Two data sets from the combined population of Swedish and Finnish Red Cattle were used to test the influence of these factors on the accuracy of imputation. Data set 1 consisted of 2,931 reference bulls and 971 test bulls, and was used for validation of imputation from 3,000 markers (3K) to 54,000 markers $(54 \mathrm{~K})$. Data set 2 contained 341 bulls in the reference set and 117 in the test set, and was used for validation of imputation from $54 \mathrm{~K}$ to high density $[777,000$ markers $(777 \mathrm{~K})]$. Both test sets were divided into 4 groups according to their relationship to the reference population. Five imputation methods (Beagle, IMPUTE2, findhap, AlphaImpute, and FImpute) were used in this study. Imputation accuracy was measured as the allele correct rate and correlation between imputed and true genotypes. Results demonstrated that the accuracy was lower when imputing from $3 \mathrm{~K}$ to $54 \mathrm{~K}$ than from $54 \mathrm{~K}$ to $777 \mathrm{~K}$. Using various imputation methods, the allele correct rates varied from 93.5 to 97.1\% when imputing from $3 \mathrm{~K}$ to $54 \mathrm{~K}$, and from 97.1 to $99.3 \%$ when imputing from $54 \mathrm{~K}$ to $777 \mathrm{~K}$; IMPUTE2 and Beagle resulted in higher accuracies and were more robust under various conditions than the other 3 methods when imputing from $3 \mathrm{~K}$ to $54 \mathrm{~K}$. The accuracy of imputation using FImpute was similar to those results from Beagle and IMPUTE2 when imputing from $54 \mathrm{~K}$ to high density, and higher than the remaining 2 methods. The results also showed that a closer relationship between test set and reference set led to a higher accuracy for all the methods. In addition, the correct rate was higher when the minor allele frequency was lower, whereas the correlation coefficient was lower when the minor allele frequency was lower. The results indicate

\footnotetext{
Received October 28, 2012.

Accepted March 28, 2013

${ }^{1}$ Corresponding authors: peipei.ma@agrsci.dk and guosheng.su@ agrsci.dk
}

that Beagle and IMPUTE2 provide the most robust and accurate imputation accuracies, but considering computing time and memory usage, FImpute is another alternative method.

Key words: imputation, relationship, minor allele frequency

\section{INTRODUCTION}

Analyses based on genomic data such as genomic selection (GS) and genome-wide association study (GWAS) has been widely used in cattle breeding. Both GS and GWAS require a large number of individuals to be genotyped with a large number of markers spread along the genome such as SNP markers (Meuwissen et al., 2001; MacLeod et al., 2010). One of the factors affecting the accuracy of genomic prediction and GWAS is the density of SNP markers (Habier et al., 2009; Meuwissen, 2009). In principle, higher density should lead to better prediction and more accurate QTL mapping, because of stronger linkage disequilibrium (LD) between markers and causative mutations. However, higher density of markers also means higher cost of genotyping.

Currently, the Illumina BovineSNP50 BeadChip (Illumina Inc., San Diego, CA; Matukumalli et al., 2009) has been widely used for genomic prediction in dairy cattle (Hayes et al., 2009; Su et al., 2010; VanRaden and Sullivan, 2010; Lund et al., 2011). However, some countries have genotyped several bulls with the 777,000-marker (777K; high-density, HD) chip with the intention of increasing the accuracy of genomic prediction, especially for genomic prediction across breeds. Higher density increases the persistence of the LD phase among populations, which is more beneficial for genomic prediction across populations than within population (Su et al., 2012). In addition, it has been proposed to genotype more individuals with a low-density chip (e.g., Bovine3K with 2,900 markers or BovineLD with 6,909 markers; Illumina Inc.) to increase the selection intensity at low cost (Boichard et al., 2012; Wiggans et al., 2012). When different SNP chips are used 
in genomic selection, imputation of missing genotypes is an important approach to make efficient use of all available marker data. Imputation is also necessary for GS or GWAS using joint reference data from exchange of genotypes between countries, where different chips are used for genotyping animals. Even for marker data from the same chip, imputation is useful for increasing the call rate of genotyped animals.

Various methods have been developed for imputation of missing genotypes. Some methods use pedigree information, whereas others do not. For example, AlphaImpute (Hickey et al., 2012b), FImpute (Sargolzaei et al., 2011), and findhap (VanRaden et al., 2011) use pedigree information, although pedigree information is not compulsory for FImpute. These methods were developed for animals and plants, as they can efficiently use complex pedigrees. Beagle (Browning and Browning, 2009) and IMPUTE2 (Howie et al., 2009), which were developed for human genetics, usually do not use pedigree information for imputation of marker data of livestock.

Imputation of missing marker genotypes is based on available marker data from a given population. The population structure and frequencies of marker genotypes in the given population have an influence on the imputation accuracy (Druet et al., 2010; Dassonneville et al., 2011; Hickey et al., 2012a). Because of differences in algorithms and different uses of information sources, the superiority of various imputation methods may differ in different imputation scenarios. Therefore, it is necessary to find the optimal imputation method and strategy to be used in the population of interest.

It has been reported that genetic variants with low frequency play a very important role in complex traits and may have larger effects than the common variants (Manolio et al., 2009). Therefore, it is necessary to investigate the efficiency of imputing markers with low minor allele frequency (MAF).

Although several studies have already been done on imputation of missing genotypes, most of these studies dealt with imputation methods and relationships between genotyped animals for imputation from a low-density panel to the 54,000-marker $(\mathbf{5 4 K})$ panel. It is necessary to compare imputation accuracy in relation to imputation methods, relationship between genotyped animals, marker densities, and marker MAF simultaneously in a given population. A simultaneous comparison is important for assessing the effect of each single factor and the combined effect of many factors on the accuracy of imputation.

The objectives of this study were 4-fold: (1) validating the accuracy of imputation from 3,000 markers $(\mathbf{3 K})$ to $54 \mathrm{~K}$ and from $54 \mathrm{~K}$ to HD using different methods in a combined population of Swedish and Finnish
Red Cattle, (2) exploring the effect of the relationship between reference and test sets on imputation accuracy, (3) comparing the sensitivity of different imputation methods with the relatedness between reference and test population, and (4) investigating the efficiency of imputation for markers with low MAF.

\section{MATERIALS AND METHODS}

\section{Data}

Two data sets composed of bulls from Swedish and Finnish Red populations were used to validate imputation procedures in this study. These 2 populations have strong genetic links due to some bulls in common use (Brøndum et al., 2011). Data set 1 consisted of 3,902 bulls (born between 1960 and 2006) genotyped with the Illumina BovineSNP50 BeadChip (54K). There were 3,893 animals with both parents, 7 animals without dam, and 2 animals without any parent in the pedigree. Data set 2 contained a subset of data set 1 , with 458 bulls (born between 1960 and 2005) that were genotyped with both the Illumina BovineHD BeadChip (777K; HD; Illumina Inc.) and the $54 \mathrm{~K}$ chip. In this data set, 450 animals had both parents, 6 animals had no dam, and 2 animals had neither sire nor dam in the pedigree.

Two imputation scenarios with regard to marker density were investigated in this study. One was imputation from $3 \mathrm{~K}$ to $54 \mathrm{~K}$, and the other was imputation from $54 \mathrm{~K}$ to $\mathrm{HD}$. In the validation of imputation from $3 \mathrm{~K}$ to $54 \mathrm{~K}$, bulls in data set 1 were divided into a reference population and a test population by birth date so that reference bulls were born before October 1, 2001. For the test population, $3 \mathrm{~K}$ marker data were derived from the $54 \mathrm{~K}$ data by masking the markers that were not on the Illumina Bovine3K BeadChip. In the validation of imputation from $54 \mathrm{~K}$ to $\mathrm{HD}$, bulls in data set 2 were divided into a reference and a test population. The test population comprised 117 bulls born after April 1, 1999, and their 54K marker genotypes were used as test data. Furthermore, markers that were in the $54 \mathrm{~K}$ chip but not in the HD map were excluded from the test data.

For all data sets, monomorphic markers were deleted. Minor allele frequencies and deviation from HardyWeinberg equilibrium were not used for editing marker data because markers with low MAF and deviation from Hardy-Weinberg equilibrium may be meaningful in genomic selection and GWAS, and one of our objectives was to compare imputation accuracy for the markers with different MAF. In addition, markers on the $\mathrm{X}$ chromosome were excluded, because no link between sire and son for markers on the $\mathrm{X}$ chromosome 
Table 1. The number of bulls in each group of test bulls before and after deleting sires or maternal grandsires in the reference set to balance the numbers of test groups ${ }^{1}$

\begin{tabular}{|c|c|c|c|c|c|c|}
\hline \multirow[b]{3}{*}{ Group } & \multicolumn{3}{|c|}{$3 \mathrm{~K}$ to $54 \mathrm{~K}$} & \multicolumn{3}{|c|}{$54 \mathrm{~K}$ to $\mathrm{HD}$} \\
\hline & \multicolumn{2}{|c|}{ No. of bulls } & \multirow{2}{*}{$\begin{array}{l}\text { No. of } \\
\text { markers }\end{array}$} & \multicolumn{2}{|c|}{ No. of bulls } & \multirow{2}{*}{$\begin{array}{c}\text { No. of } \\
\text { markers }\end{array}$} \\
\hline & Before & After & & Before & After & \\
\hline Reference & 2,945 & \multirow[t]{2}{*}{2,931} & 45,418 & 349 & \multirow[t]{2}{*}{341} & \multirow{7}{*}{$\begin{array}{r}641,466 \\
41,383\end{array}$} \\
\hline Test $^{2}$ & 977 & & 2,657 & 117 & & \\
\hline GRPsmgs & 825 & \multicolumn{2}{|l|}{245} & 85 & 31 & \\
\hline GRPsire & 50 & \multicolumn{2}{|l|}{249} & 14 & 30 & \\
\hline GRPmgs & 74 & \multicolumn{2}{|l|}{239} & 16 & 28 & \\
\hline GRPnone & 22 & \multicolumn{2}{|l|}{244} & 2 & \multirow[t]{2}{*}{28} & \\
\hline Total & 3,902 & & & 458 & & \\
\hline \multicolumn{7}{|c|}{$\begin{array}{l}{ }^{1} 3 \mathrm{~K}=3,000 \text { markers (Illumina Bovine3K BeadChip; Illumina Inc., San Diego, CA); } 54 \mathrm{~K}=54,000 \text { markers } \\
\text { (Illumina BovineSNP50 BeadChip; Illumina Inc.); HD = high density }[777,000 \text { markers (777K); Illumina } \\
\text { BovineHD BeadChip; Illumina Inc. } .\end{array}$} \\
\hline
\end{tabular}

may influence imputation accuracy differently when using different imputation methods. After filtering, in data set 1, 45,418 markers were available in the $54 \mathrm{~K}$ data and 2,657 in the mimicked 3K chip. And in data set 2, 641,466 markers were available in the HD data and 41,383 in the $54 \mathrm{~K}$ test data.

To investigate the effects of relationship between reference population and test population on imputation accuracy, the test populations were divided into 4 groups: (1) both the sire and maternal grandsire (MGS) of the bulls were in the reference population $\left(\mathbf{G R P}_{\text {smgs }}\right),(2)$ only the sire was in the reference population $\left(\mathbf{G R P}_{\text {sire }}\right)$, (3) only the MGS was in the reference population $\left(\mathbf{G R P}_{\mathbf{m g s}}\right)$, and (4) neither the sire nor the MGS was in the reference population $\left(\mathbf{G R P}_{\text {none }}\right)$. To make the number of animals in each group even, 14 bulls in the reference population were removed from the $54 \mathrm{~K}$ reference data, and 8 bulls were excluded from the HD reference data to eliminate genetic links with some animals in the test set. This was done by an iterative procedure that minimized the difference between the sizes of the 4 groups and the number of bulls to be deleted. In each iteration, a chi-squared value for each sire or MGS in the reference population at the current stage was calculated according to observed number and expected number (total number of test animals divided by 4), given that this sire or MGS was excluded from the reference population. Then, the sire or MGS with the lowest chi-squared value was deleted from the reference population. The iteration procedure was repeated until a minimum chi-squared value was achieved. The number of animals in each test group before and after deleting the animals from the reference is shown in Table 1.

\section{Imputation}

Missing marker genotypes were imputed using Beagle version 3.3.1 (Browning and Browning, 2009), findhap version 2 (VanRaden et al., 2011), FImpute version 2.2 (Sargolzaei et al., 2011), AlphaImpute version 1.16 (Hickey et al., 2012b), and IMPUTE2 version 2.2.2 (Howie et al., 2009). Beagle (Browning and Browning, 2007) uses a hidden Markov model to predict the missing genotypes. A direct acyclic graph is constructed by summarizing haplotypes in the reference population. The program findhap (VanRaden et al., 2011) uses both family and population information. The haplotype library, which is sorted by frequency, is constructed with a certain number of SNP in the reference population. The haplotypes in the test population are searched in the haplotype library. FImpute (Sargolzaei et al., 2011) uses 3 steps to impute the missing genotypes. First, pedigree information is used to impute the missing genotypes that can be inferred with high certainty. Second, haplotypes are constructed with population information based on a sliding window approach. Third, the missing genotypes are filled in according to the haplotypes constructed in the second step. AlphaImpute (Hickey et al., 2012b) uses segregation analysis and haplotype library imputation. The allele probabilities are calculated using segregation analysis. The genotypes in all genotyped animals are phased using long-range phasing and haplotype library imputation. The missing genotypes are filled in by matching the allelic probabilities to the haplotypes. The program IMPUTE2 (Howie et al., 2009) uses a hidden Markov model to model the haplotypes underlying genotypes to mosaic blocks in the reference panel, and then imputes the missing 
Table 2. The proportion of imputed markers in each minor allele frequency (MAF) bin ${ }^{1}$

\begin{tabular}{lcc}
\hline MAF bin & $\begin{array}{c}\text { Ratio } \\
(3 \mathrm{~K} \text { to } 54 \mathrm{~K})\end{array}$ & $\begin{array}{c}\text { Ratio } \\
(54 \mathrm{~K} \text { to HD })\end{array}$ \\
\hline$[0,0.025]$ & 0.09 & 0.06 \\
{$[0.025,0.05]$} & 0.05 & 0.04 \\
{$[0.05,0.075]$} & 0.05 & 0.04 \\
{$[0.075,0.1]$} & 0.05 & 0.04 \\
{$[0.1,0.2]$} & 0.19 & 0.17 \\
{$[0.2,0.3]$} & 0.19 & 0.20 \\
{$[0.3,0.4]$} & 0.19 & 0.22 \\
{$[0.4,0.5]$} & 0.19 & 0.23 \\
\hline
\end{tabular}

${ }^{1} 3 \mathrm{~K}=3,000$ markers (Illumina Bovine3K BeadChip; Illumina Inc., San Diego, CA); $54 \mathrm{~K}=54,000$ markers (Illumina BovineSNP50 BeadChip; Illumina Inc.); HD = high density [777,000 markers (777K); Illumina BovineHD BeadChip; Illumina Inc.].

genotypes in the test panel by choosing the most similar subset to the previous haplotype estimates of the individual. A detailed description on the algorithms for these methods was presented by Sun et al. (2012).

Pedigree information was provided for AlphaImpute, findhap, and FImpute. The pedigree was built by tracing the ancestral pedigree of the genotyped bulls back as many generations as possible. In total, the numbers of individuals were 23,575 in the pedigree file for data set 1 and 4,266 in the pedigree for data set 2. No pedigree was used in Beagle and IMPUTE2, although Beagle can use parent-offspring trio or duo information. The marker data were divided into single chromosomes, and imputation was performed for each chromosome separately. When using IMPUTE2, the effective population size was set to 100 . The whole chromosome was imputed directly for data set 1 , whereas each chromosome was divided into several segments of $6 \mathrm{Mb}$ for data set 2, as recommended by the authors of IMPUTE2, considering the computational efficiency. When using AlphaImpute, editing parameters were set to 95.0, 5.0, and 98.0. These parameters control the internal editing to separate the high-density-group animals and low-density-group animals. First, the animals that are genotyped for more than $95 \%$ of the SNP enter the high-density group. Second, the markers in this preselected high-density group are filtered, with a maximum missing threshold of $5.0 \%$. Third, the animals that are genotyped more than $98.0 \%$ for the remaining markers in the second step enter the final high-density group. The CoreLengths and CoreAndTailLengths parameters, which are used to control the number of markers in a phasing run, were set to 10 times the default values when imputing from $54 \mathrm{~K}$ to HD. For Beagle, findhap, and FImpute, the recommended parameters were used.

The most likely genotypes were used to validate the imputation accuracy. It was observed that Beagle, FImpute, and IMPUTE2 imputed all the missing genotypes, and findhap imputed almost all missing geno- types in the current data. However, there was still a large number of missing genotypes $(16.9 \%$ for data set 1 and $9.0 \%$ for data set 2) after imputation when using AlphaImpute. Therefore, the remaining missing genotypes in the output from AlphaImpute were filled in by 2 different methods: (1) replacement with the most likely genotypes, which were obtained by rounding the expectation $E=\pi_{0} \times 0+\pi_{1} \times 1+\pi_{2} \times 2$, where $\pi_{0}$, $\pi_{1}$, and $\pi_{2}$ were the probability of the genotype being 0,1 , and 2 (representing $\mathrm{A}_{1} \mathrm{~A}_{1}, \mathrm{~A}_{1} \mathrm{~A}_{2}$, and $\mathrm{A}_{2} \mathrm{~A}_{2}$, respectively) - for example, the most likely genotype was 2 if $E=1.85$, but 1 if $E=1.35$; and (2) further imputation using Beagle (AlphaBea).

\section{Validation}

Two validation criteria were used to measure the accuracy of imputation in this study. One was the allele correct rate $(\mathbf{C R})$, which was calculated as the number of correctly imputed alleles divided by the total number of imputed alleles in the test data set. The other was the correlation coefficient between the original and the imputed genotypes, which were recorded as 0,1 , and 2 for genotypes $A_{1} A_{1}, A_{1} A_{2}$, and $A_{2} A_{2}$, respectively. The validation was carried out for each group of test bulls separately, and the pooled accuracy was calculated using the whole test data set. The allele CR and correlation coefficient were calculated across all imputed loci. In addition, to detect the association between accuracy of imputation and MAF, the markers were classified into 8 bins according to MAF: [0, 0.025], [0.025, 0.05], $[0.05,0.075],[0.075,0.1],[0.1,0.2],[0.2,0.3],[0.3,0.4]$, and $[0.4,0.5]$. The proportion of markers in each bin is shown in Table 2. The accuracy of imputation was calculated for each bin.

As QTL may tend to be linked with rare alleles (Manolio et al., 2009; Hickey et al., 2012a), it is necessary to test the efficiency of imputation for alleles at different frequencies. The alleles were divided into 10 bins, according to their frequency, with an increment of 0.1 . The CR for each of the 2 alleles at a locus was calculated separately. Thus, the CR of an allele at a locus was calculated as the proportion of correctly imputed allele counts on the total counts of this allele. The CR for a bin was the average CR of the alleles in this bin.

An alternative to allele CR was genotype CR. Similar to allele CR, which measures how well the alleles are imputed, genotype CR measures how well the genotypes are imputed. Genotype CR was calculated as the number of correctly imputed genotypes divided by the total number of imputed genotypes in the test data set. As the measures of genotype CR was highly consistent with allele CR, results of the genotype CR will be presented only briefly. 
Table 3. Allele correct rate for imputation from 3,000 markers (3K; Illumina Bovine3K BeadChip; Illumina Inc., San Diego, CA) to 54,000 markers (54K; Illumina BovineSNP50 BeadChip; Illumina Inc.)

\begin{tabular}{|c|c|c|c|c|c|c|}
\hline \multirow[b]{2}{*}{ Test group ${ }^{1}$} & \multicolumn{6}{|c|}{ Imputation method ${ }^{2}$} \\
\hline & Beagle & findhap & AlphaImpute ${ }^{3}$ & AlphaBea $^{4}$ & FImpute & IMPUTE2 \\
\hline GPsmgs & 96.9 & 95.6 & 95.4 & 96.9 & 96.6 & 97.8 \\
\hline GPsire & 96.6 & 95.1 & 95.0 & 96.3 & 96.3 & 97.5 \\
\hline GPmgs & 96.2 & 92.8 & 92.9 & 94.3 & 94.4 & 97.0 \\
\hline GRPnone & 95.4 & 91.2 & 91.7 & 93.0 & 93.5 & 96.2 \\
\hline Pooled & 96.3 & 93.7 & 93.7 & 95.1 & 95.2 & 97.1 \\
\hline Missing & 0 & 0.08 & 0 & 0 & 0 & 0 \\
\hline
\end{tabular}

${ }^{1}$ The test population was divided into 4 groups according to whether their sire and (or) maternal grandsire (MGS) were in the reference population. GRPsmgs = both sire and MGS; GRPsire = only sire; GRPmgs = only MGS; GRPnone = neither sire nor MGS.

${ }^{2}$ Imputation methods used: Beagle (Browning and Browning, 2009), findhap (VanRaden et al., 2011), AlphaImpute (Hickey et al., 2012b), FImpute (Sargolzaei et al., 2011), and IMPUTE2 (Howie et al., 2009).

${ }^{3}$ The values were the rounded expected genotypes of AlphaImpute (e.g., the value used for comparison was 2 when the expected genotype was $\geq 1.5$ ).

${ }^{4}$ The missing genotypes after imputed by AlphaImpute were imputed by Beagle.

\section{RESULTS}

\section{Accuracy of Imputation from $3 K$ to $54 K$}

The CR and correlations for imputation from $3 \mathrm{~K}$ to $54 \mathrm{~K}$ are shown in Table 3 and Table 4, respectively. As shown in Table 3, the CR of imputation using different approaches for the 4 test groups ranged from 91.2 to $97.8 \%$. According to the CR, the rank of the imputation approaches in descending order was IMPUTE2, Beagle, FImpute, AlphaBea, and the last were AlphaImpute and findhap.

In all approaches, CR increased with increasing relatedness between the reference and the test population. However, the influence of relatedness on $\mathrm{CR}$ was more profound for findhap, AlphaImpute, and FImpute which used pedigree information. Thus, the difference between $\mathrm{CR}$ of $\mathrm{GRP}_{\text {smgs }}$ and $\mathrm{GRP}_{\text {none }}$ was $4.4 \%$ for findhap, 3.9\% for AlphaBea, 3.8\% for AlphaImpute, $3.2 \%$ for FImpute, $1.6 \%$ for IMPUTE2, and $1.5 \%$ for Beagle. Moreover, the difference between CR of the 5 imputation approaches was larger for the animals without close relatives in the reference population than the animals with close relatives.

When accuracy of imputation was measured as the correlation coefficient between imputed and original genotypes, the rank of the imputation approaches was almost the same as the rank based on CR (Table 4). The only difference was that the correlation for FImpute was lower than for AlphaBea. Moreover, the differences between the correlation coefficients from different imputation approaches were larger than the differences between CR. The highest correlation was 0.886 when using IMPUTE2 and lowest 0.750 when using findhap for GRPsmgs, and 0.853 and 0.656 for GRPnone.

Table 4. Correlation coefficients for imputation from 3,000 markers (3K; Illumina Bovine3K BeadChip; Illumina Inc., San Diego, CA) to 54,000 markers (54K; Illumina BovineSNP50 BeadChip; Illumina Inc.)

\begin{tabular}{lcccccc}
\hline & \multicolumn{5}{c}{ Imputation method $^{2}$} \\
\cline { 2 - 6 } Test group $^{1}$ & Beagle & findhap & AlphaImpute $^{3}$ & AlphaBea $^{4}$ & FImpute & IMPUTE2 \\
\cline { 2 - 6 } GRPsmgs & 0.896 & 0.835 & 0.829 & 0.887 & 0.866 & 0.917 \\
GRPsire & 0.885 & 0.811 & 0.813 & 0.865 & 0.855 & 0.903 \\
GRPmgs & 0.867 & 0.717 & 0.731 & 0.792 & 0.783 & 0.883 \\
GRPnone & 0.832 & 0.656 & 0.685 & 0.739 & 0.740 & 0.853 \\
Pooled & 0.866 & 0.750 & 0.760 & 0.819 & 0.807 & 0.886 \\
\hline
\end{tabular}

${ }^{1}$ The test population was divided into 4 groups according to whether their sire and (or) maternal grandsire (MGS) were in the reference population. GRPsmgs = both sire and MGS; GRPsire = only sire; GRPmgs = only MGS; GRPnone = neither sire nor MGS

${ }^{2}$ Imputation methods used: Beagle (Browning and Browning, 2009), findhap (VanRaden et al., 2011), AlphaImpute (Hickey et al., 2012b), FImpute (Sargolzaei et al., 2011), and IMPUTE2 (Howie et al., 2009).

${ }^{3}$ The values were the rounded expected genotypes of AlphaImpute (e.g., the value used for comparison was 2 when the expected genotype was $\geq 1.5$ ).

${ }^{4}$ The missing genotypes after imputed by AlphaImpute were imputed by Beagle. 
Table 5. Allele correct rate for imputation from 54,000 markers (54K; Illumina BovineSNP50 BeadChip; Illumina Inc., San Diego, CA) to high density [HD; 777,000 markers (777K); Illumina BovineHD BeadChip; Illumina Inc.]

\begin{tabular}{lcccccc}
\hline & \multicolumn{5}{c}{ Imputation method $^{2}$} \\
\cline { 2 - 6 } Test group $^{1}$ & Beagle & findhap & AlphaImpute $^{3}$ & AlphaBea $^{4}$ & FImpute & IMPUTE2 $^{2}$ \\
\hline GRPsmgs & 99.4 & 99.2 & 98.8 & 99.1 & 99.6 & 99.6 \\
GRPsire & 99.1 & 98.1 & 98.1 & 98.5 & 99.2 & 99.3 \\
GRPmgs & 99.1 & 97.7 & 96.1 & 97.1 & 99.2 & 99.3 \\
GRPnone & 98.7 & 95.7 & 95.6 & 96.6 & 98.9 & 99.0 \\
Pooled & 99.1 & 97.6 & 97.1 & 97.8 & 99.2 & 99.3 \\
Missing & 0.0 & 0.0 & 0 & 0.0 & 0.0 & 0.0 \\
\hline
\end{tabular}

${ }^{1}$ The test population was divided into 4 groups according to whether their sire and (or) maternal grandsire (MGS) were in the reference population. GRPsmgs $=$ both sire and MGS; GRPsire = only sire; GRPmgs $=$ only MGS; GRPnone = neither sire nor MGS.

${ }^{2}$ Imputation methods used: Beagle (Browning and Browning, 2009), findhap (VanRaden et al., 2011), AlphaImpute (Hickey et al., 2012b), FImpute (Sargolzaei et al., 2011), and IMPUTE2 (Howie et al., 2009).

${ }^{3}$ The values were the rounded expected genotypes of AlphaImpute (e.g., the value used for comparison was 2 when the expected genotype was $\geq 1.5$ ).

${ }^{4}$ The missing genotypes after imputed by AlphaImpute were imputed by Beagle.

\section{Accuracy of Imputation from $54 \mathrm{~K}$ to $H D$}

As shown in Table 5 and Table 6, imputation from $54 \mathrm{~K}$ to HD generally gave higher accuracies and smaller differences between the approaches, compared with imputation from $3 \mathrm{~K}$ to $54 \mathrm{~K}$. In general, the rank of the imputation approaches in descending order was IMPUTE2, FImpute, Beagle, AlphaBea, findhap, and AlphaImpute. The difference using different methods was smallest in GRPsmgs (the highest vs. the lowest were $99.6 \%$ vs. $98.8 \%$ in CR, and 0.988 vs. 0.972 in correlation), and largest in GRPnone (the highest vs. the lowest were $99.0 \%$ vs. $97.1 \%$ in CR, and 0.975 vs. 0.909 in correlation).

Similar to imputation from $3 \mathrm{~K}$ to $54 \mathrm{~K}$, relatedness between test bulls and reference bulls had an influence on the accuracy of imputation, and the influence was larger when using findhap and AlphaImpute than when using IMPUTE2, FImpute, and Beagle. Comparing $\mathrm{GRP}_{\text {smgs }}$ with $\mathrm{GRP}_{\text {none }}$, the difference in CR was 3.5, $3.2,2.5,0.7,0.7$, and $0.6 \%$ for findhap, AlphaImpute, AlphaBea, FImpute, Beagle, and IMPUTE2, respectively.

\section{Effect of MAF on Imputation Accuracy}

Figures 1, 2, 3, and 4 present $\mathrm{CR}$ and correlation coefficients for the 8 bins of markers according to MAF, calculated by pooling the data of the 4 test groups. Correct rates and correlation coefficients in relation to MAF had different patterns. Correct rate was higher when MAF was lower (Figures 1 and 3). In contrast,

Table 6. Correlation coefficients for imputation from 54,000 markers (54K; Illumina BovineSNP50 BeadChip; Illumina Inc., San Diego, CA) to high density [HD; 777,000 markers (777K); Illumina BovineHD BeadChip; Illumina Inc.]

\begin{tabular}{lcccccc}
\hline & \multicolumn{5}{c}{${\text { Imputation } \text { method }^{2}}$} \\
\cline { 2 - 6 } Test group $^{1}$ & Beagle & findhap & AlphaImpute $^{3}$ & AlphaBea $^{4}$ & FImpute $^{\text {IMPUTE2 }}$ \\
\hline GRPsmgs & 0.982 & 0.972 & 0.963 & 0.971 & 0.987 & 0.988 \\
GRPsire & 0.970 & 0.948 & 0.941 & 0.952 & 0.976 & 0.978 \\
GRPmgs & 0.971 & 0.925 & 0.883 & 0.911 & 0.973 & 0.977 \\
GRPnone & 0.956 & 0.892 & 0.865 & 0.892 & 0.962 & 0.966 \\
Pooled & 0.967 & 0.925 & 0.909 & 0.927 & 0.972 & 0.975 \\
\hline
\end{tabular}

${ }^{1}$ The test population was divided into 4 groups according to whether their sire and (or) maternal grandsire (MGS) were in the reference population. GRPsmgs = both sire and MGS; GRPsire = only sire; GRPmgs = only MGS; GRPnone = neither sire nor MGS.

${ }^{2}$ Imputation methods used: Beagle (Browning and Browning, 2009), findhap (VanRaden et al., 2011), AlphaImpute (Hickey et al., 2012b), FImpute (Sargolzaei et al., 2011), and IMPUTE2 (Howie et al., 2009 ).

${ }^{3}$ The values were the rounded expected genotypes of AlphaImpute (e.g., the value used for comparison was 2 when the expected genotype was $\geq 1.5$ ).

${ }^{4}$ The missing genotypes after imputed by AlphaImpute were imputed by Beagle. 


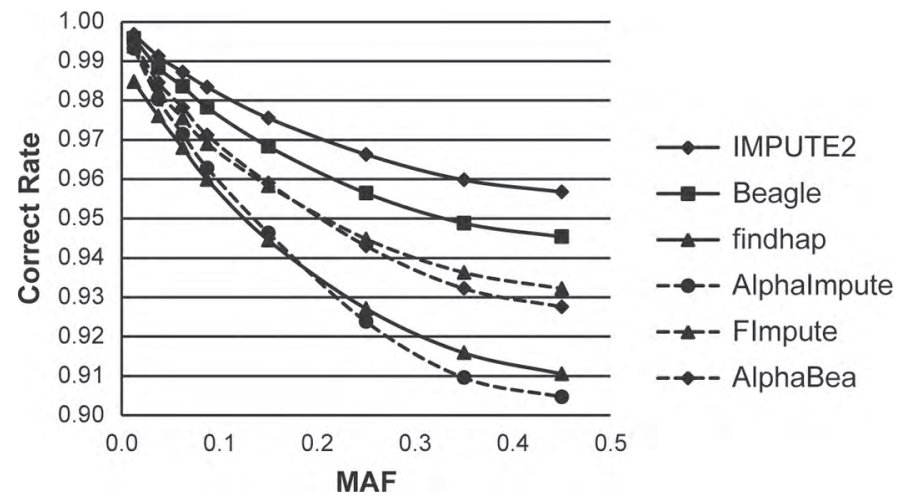

Figure 1. Correct rate in relation to minor allele frequency (MAF) for imputation from 3,000 markers (3K; Illumina Bovine3K BeadChip; Illumina Inc., San Diego, CA) to 54,000 markers (54K; Illumina BovineSNP50 BeadChip; Illumina Inc.) using different imputation methods. Imputation methods used: IMPUTE2 (Howie et al., 2009), Beagle (Browning and Browning, 2009), findhap (VanRaden et al., 2011), AlphaImpute (Hickey et al., 2012b), and FImpute (Sargolzaei et al., 2011). AlphaBea means that the missing genotypes after imputation by AlphaImpute were imputed by Beagle.

correlation coefficient was lower when MAF was lower (Figures 2 and 4).

The trends of $\mathrm{CR}$ and correlation coefficient in relation to MAF were the same for all approaches. However, the approaches that used pedigree information were more sensitive to changes in MAF. In addition, the changes of $\mathrm{CR}$ and correlation coefficient with MAF were larger in imputation from $3 \mathrm{~K}$ to $54 \mathrm{~K}$ than in imputation from $54 \mathrm{~K}$ to $\mathrm{HD}$.

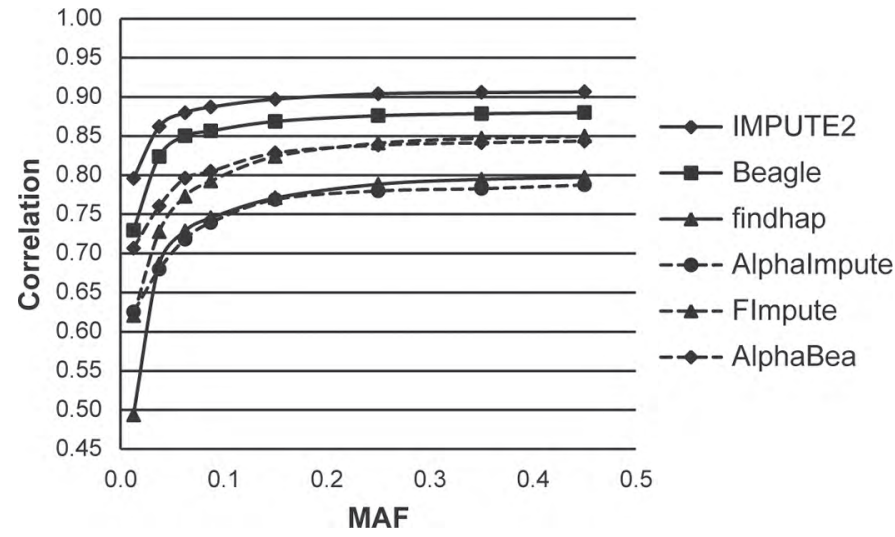

Figure 2. Correlation in relation to minor allele frequency (MAF) for imputation from 3,000 markers (3K; Illumina Bovine3K BeadChip; Illumina Inc., San Diego, CA) to 54,000 markers (54K; Illumina BovineSNP50 BeadChip; Illumina Inc.) using different imputation methods. Imputation methods used: IMPUTE2 (Howie et al., 2009), Beagle (Browning and Browning, 2009), findhap (VanRaden et al., 2011), AlphaImpute (Hickey et al., 2012b), and FImpute (Sargolzaei et al., 2011). AlphaBea means that the missing genotypes after imputation by AlphaImpute were imputed by Beagle.

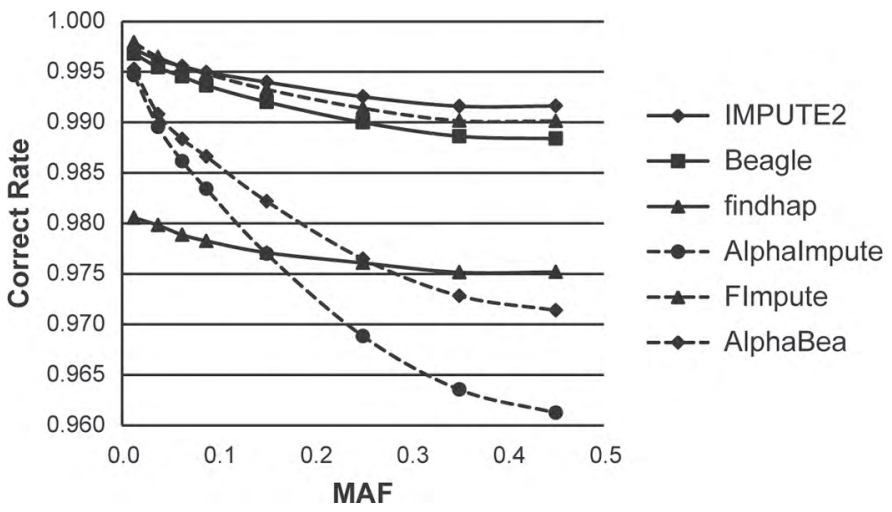

Figure 3. Correct rate in relation to minor allele frequency (MAF) for imputation from 54,000 markers (54K; Illumina BovineSNP50 BeadChip; Illumina Inc., San Diego, CA) to high density [HD; 777,000 markers (777K); Illumina BovineHD BeadChip; Illumina Inc.] using different imputation methods. Imputation methods used: IMPUTE2 (Howie et al., 2009), Beagle (Browning and Browning, 2009), findhap (VanRaden et al., 2011), AlphaImpute (Hickey et al., 2012b), and FImpute (Sargolzaei et al., 2011). AlphaBea means that the missing genotypes after imputation by AlphaImpute were imputed by Beagle.

Based on the results from imputation using IMPUTE2, allele CR for each allele (instead of CR for each locus) was calculated separately. As shown in Figure 5, $\mathrm{CR}$ increased with increasing allele frequency, indicating that rare alleles were more difficult to impute than common alleles.

\section{Genotype Correct Rate}

Genotype CR was used as an alternative measure of imputation accuracy. It was observed that the geno-

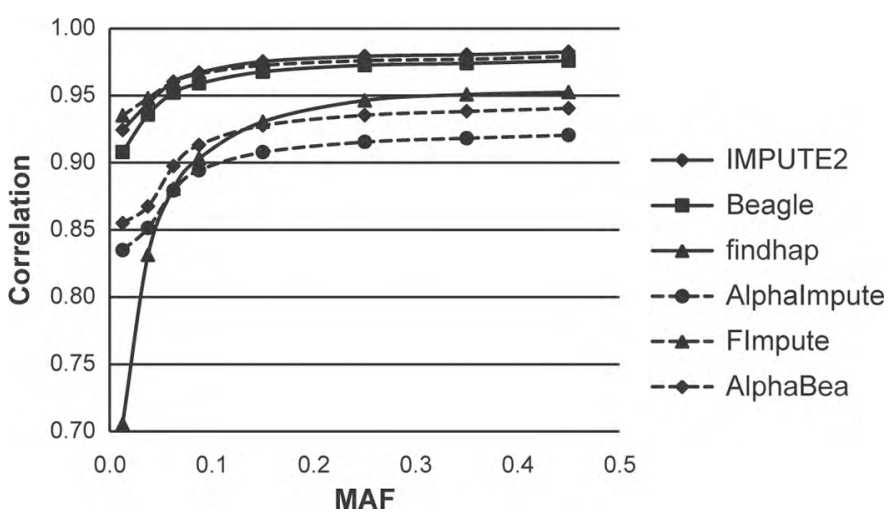

Figure 4. Correlation in relation to minor allele frequency (MAF) for imputation from 54,000 markers (54K; Illumina BovineSNP50 BeadChip; Illumina Inc., San Diego, CA) to high density [HD; 777,000 markers (777K); Illumina BovineHD BeadChip; Illumina Inc.] using different imputation methods. Imputation methods used: IMPUTE2 (Howie et al., 2009), Beagle (Browning and Browning, 2009), findhap (VanRaden et al., 2011), AlphaImpute (Hickey et al., 2012b), and FImpute (Sargolzaei et al., 2011). AlphaBea means that the missing genotypes after imputation by AlphaImpute were imputed by Beagle. 


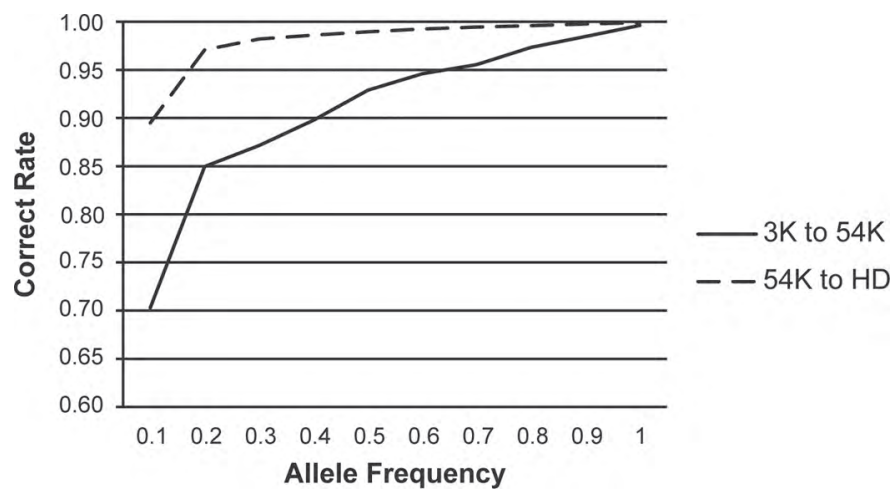

Figure 5. Allele correct rate in relation to allele frequency, calculated from imputation for chromosome 28 using IMPUTE2 (Howie et al., 2009). 3K = 3,000 markers (Illumina Bovine3K BeadChip; Illumina Inc., San Diego, CA); 54K = 54,000 markers (Illumina BovineSNP50 BeadChip; Illumina Inc.); HD = high density [777,000 markers (777K); Illumina BovineHD BeadChip; Illumina Inc.].

type error rate was highly consistent with allele CR. In other words, genotype error rate $(1-$ genotype CR) was twice or almost twice as much as allele error rate $(1$ - allele CR $)$ in all scenarios. For example, when using FImpute for imputation from $3 \mathrm{~K}$ to $54 \mathrm{~K}$, the allele correct rate for GRPsmgs was $96.6 \%$ and the genotype correct rate was $93.3 \%$. The differences between 2 times the allele error rate and genotype error rate in relation to $\operatorname{MAF}(2 \times$ allele error rate - genotype error rate $)$ are shown in Figure 6. When the MAF became higher, the difference became larger. This indicated that the probability of imputing a homozygous genotype to the other homozygous genotype was higher for markers with higher MAF.

\section{Computational Demands}

Table 7 presents the time and random access memory (RAM) used for imputation on chromosome 1. All the jobs were run on a Linux server (http://www.linux. $\mathrm{org} /$ ) with an $\operatorname{Intel}(\mathrm{R}) \mathrm{Xeon}(\mathrm{R})$ central processing unit (CPU) E5450 (Intel Corp., Santa Clara, CA) at 3.00 $\mathrm{GHz}$, with a total RAM of $32 \mathrm{~GB}$. One single job was run on this server at a given time. In the imputation from $3 \mathrm{~K}$ to $54 \mathrm{~K}, 2,931$ bulls were in the reference population and 971 in the test population. In the imputation from $54 \mathrm{~K}$ to $\mathrm{HD}, 341$ bulls were in the reference population and 117 in the test population. In both scenarios, AlphaImpute took the longest time and used the largest memory (peak memory), whereas findhap and FImpute took the shortest time and FImpute also used the least memory.

\section{DISCUSSION}

This study investigated accuracy of imputation using different approaches, based on different marker data sets from the Finnish and Swedish Red Cattle populations. The results indicate that the accuracy of imputation depends on marker density, imputation methods, relatedness between test population and reference population, and MAF of the markers.

\section{Marker Density}

The accuracy of imputation from $54 \mathrm{~K}$ to HD was higher than the accuracy from $3 \mathrm{~K}$ to $54 \mathrm{~K}$. The results were consistent with previous studies (Dassonneville et al., 2011; Brøndum et al., 2012; Sun et al., 2012). It was reported that the error rate $(1-\mathrm{CR})$ was $5.5 \%$ when imputing from $3 \mathrm{~K}$ to $54 \mathrm{~K}$ in Nordic Holstein (Dassonneville et al., 2011) and $0.77 \%$ when imputing from $54 \mathrm{~K}$ to HD in the same population ( $\mathrm{Su}$ et al., 2012). It was reported that the density of the markers in the test panel was an important factor affecting imputation accuracy (Hickey et al., 2012a).

According to the results from this study, the $54 \mathrm{~K}$ panel can be accurately imputed to the HD panel. Using IMPUTE2, Beagle and FImpute, the CR was over $99 \%$ in the current population. However, the imputation from $3 \mathrm{~K}$ to $54 \mathrm{~K}$ panel is not satisfactory. The highest CR were 97.1 and $96.3 \%$ when using IMPUTE2 and Beagle, respectively. It has been reported that imputation accuracy has a large influence on the reliability of genomic prediction. Brøndum et al. (2011) imputed $3 \mathrm{~K}$ to $54 \mathrm{~K}$ panel in Nordic Holstein cattle using a combination of the DagPhase program (Druet and Georges, 2010) and Beagle and reported that the error rate for the imputation was $3.9 \%$, and the corresponding decrease in reliability of genomic prediction was 5\%. Segelke et al. (2012) used findhap and Beagle to impute mimicked $3 \mathrm{~K}$ genotypes to the $54 \mathrm{~K}$ SNP panel. The error rates of imputation with findhap and Beagle

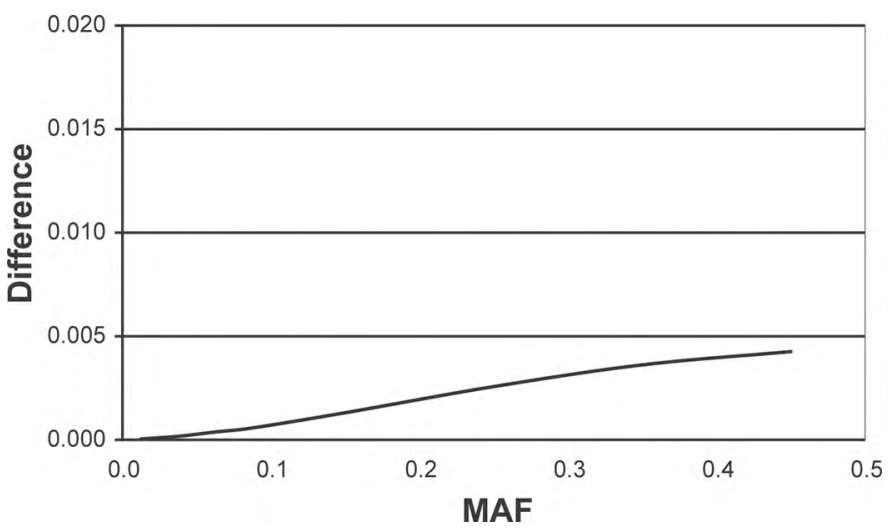

Figure 6. The difference between 2 times the allele error rate and genotype error rate in relation to minor allele frequency (MAF; $2 \times$ allele error rate - genotype error rate). 
Table 7. Time and memory used for imputation on chromosome 1

\begin{tabular}{llrlrr}
\hline & \multicolumn{2}{c}{$3 \mathrm{~K}$ to $54 \mathrm{~K}^{2}$} & & \multicolumn{2}{c}{$54 \mathrm{~K}$ to $\mathrm{HD}^{3}$} \\
\cline { 2 - 3 } \cline { 5 - 6 } Imputation method $^{1}$ & Time & Memory $(\mathrm{kB})$ & & Time & Memory $(\mathrm{kB})$ \\
\hline Beagle & $7 \mathrm{~h}, 19 \mathrm{~m}, 30 \mathrm{~s}$ & $4,849,076$ & & $39 \mathrm{~m}, 42 \mathrm{~s}$ & $1,959,832$ \\
findhap & $1 \mathrm{~m}, 45 \mathrm{~s}$ & 190,896 & & $1 \mathrm{~m}, 24 \mathrm{~s}$ & 426,264 \\
AlphaImpute & $21 \mathrm{~h}, 20 \mathrm{~m}, 55 \mathrm{~s}$ & $10,490,548$ & & $22 \mathrm{~h}, 37 \mathrm{~m}, 32 \mathrm{~s}$ & $15,524,772$ \\
FImpute & $1 \mathrm{~m}, 51 \mathrm{~s}$ & 44,544 & & $1 \mathrm{~m}, 48 \mathrm{~s}$ & 80,096 \\
IMPUTE2 & $8 \mathrm{~h}, 19 \mathrm{~m}, 2 \mathrm{~s}^{4}$ & 274,880 & & $9 \mathrm{~h}, 01 \mathrm{~m}, 19 \mathrm{~s}^{5}$ & 88,052 \\
\hline
\end{tabular}

${ }^{1}$ Imputation methods used: Beagle (Browning and Browning, 2009), findhap (VanRaden et al., 2011), AlphaImpute (Hickey et al., 2012b), FImpute (Sargolzaei et al., 2011), and IMPUTE2 (Howie et al., 2009).

${ }^{2} 3 \mathrm{~K}=3,000$ markers (Illumina Bovine3K BeadChip; Illumina Inc., San Diego, CA); 54K = 54,000 markers (Illumina BovineSNP50 BeadChip; Illumina Inc.). Number of bulls in the reference $=2,931$; number of bulls in the test $=971$.

${ }^{3} \mathrm{HD}=$ high density [777,000 markers (777K); Illumina BovineHD BeadChip; Illumina Inc.]. Number of bulls in the reference $=341$; number of bulls in the test $=117$.

${ }^{4}$ The whole chromosome was imputed as 1 segment.

${ }^{5}$ The chromosome was divided into 27 segments by the interval of $6 \mathrm{MB}$. This time was the sum of all the 27 segments.

were 3.3 and $1.6 \%$, respectively. The corresponding decreases in reliability of genomic prediction (average for 12 traits) were 5.3 and $2.6 \%$. Based on results by the 2 previously mentioned studies, Su et al. (2012) declared that per $1 \%$ of imputation allele error rate the resulting loss in reliability of genomic prediction was 1.3 percentage points. Mulder et al. (2012) derived the relationship between the imputation accuracy (measured as correlation) and the accuracy of direct genomic value, and investigated it empirically. Their results showed the accuracy of direct genomic value was increased linearly with imputation accuracy. The above information suggests that the $3 \mathrm{~K}$ chip is not the optimal in the current population (a mixture of Finnish Red population and Swedish Red population). Alternatively, a new low-density chip [BovineLD; 7,000 markers $(\mathbf{7 K})$ ] was assessed and reported to give better accuracy (Boichard et al., 2012; Dassonneville et al., 2012) than the 3K chip. Therefore, it may be a good choice to use the $7 \mathrm{~K}$ chip in this population.

\section{Imputation Methods}

Five imputation methods were used in this study. Methods IMPUTE2 and Beagle do not use and require pedigree information explicitly but the 2 methods are actually able to use family information. The individuals in the test data should share longer segment with a recent ancestor in the reference data when using Beagle (Browning and Browning, 2009). Method IMPUTE2 uses surrogate family information based on the genomic information (Howie et al., 2011). It was found that IMPUTE2 and Beagle performed better in both data sets from the current population. Method FImpute performed as well as IMPUTE2 and Beagle in imputation from $54 \mathrm{~K}$ to $\mathrm{HD}$ but not in imputation from $3 \mathrm{~K}$ to $54 \mathrm{~K}$. The results were not consistent with those reported in the study of Johnston et al. (2011), where FImpute outperformed Beagle for the imputation from $3 \mathrm{~K}$ to $54 \mathrm{~K}$ in a Holstein population and a Brown Swiss population. One possible reason could be that the present population was a combination of 2 Red cattle populations. However, a previous study showed a strong genetic link between Finish and Swedish Red populations (Brøndum et al., 2011) and combined Finish and Swedish Red reference improved the imputation accuracy for imputation from 54K to HD using Beagle (Brøndum et al., 2012). The other reason could be that the relationship between individuals was stronger and the number of genotyped animals was much larger in their population than in the present population, and their genotyped animals included females. In a previous study (Sun et al., 2012) on imputation in Angus cattle, it was reported that Beagle performed best for imputation from $5 \mathrm{~K}$ to $50 \mathrm{~K}$. However, contrary to the present study, IMPUTE2 performed worse than FImpute and findhap. The reason could be that each chromosome was split into several segments when using IMPUTE2 in their study, whereas the whole chromosome was used for imputation from $3 \mathrm{~K}$ to $54 \mathrm{~K}$ in the present study. When splitting a chromosome into several segments, the LD information between these segments cannot be used for imputation; consequently, the accuracy of imputation decreases. Splitting a chromosome into several segments is usually applied in imputation of high-density markers to avoid a high computational demand.

As a whole, IMPUTE2 and Beagle performed better than the other methods for imputation from $3 \mathrm{~K}$ to $54 \mathrm{~K}$ in the current population, and FImpute performed as 
well as IMPUTE2 and Beagle for imputation from 54K to HD, but used much less time. The disadvantage of IMPUTE2 and Beagle is long running time and large memory usage. The running time will become a very important issue with more and more animals being genotyped in the future. In practical genomic evaluation, it could be a feasible approach to store the established haplotypes for imputing new marker data, and update the haplotypes once per year. An additional trial showed that the running time for Beagle was almost halved when imputing $54 \mathrm{~K}$ to HD based on previously phased HD haplotypes. It was reported that the running time was reduced from $31 \mathrm{~h}$ to less than $1 \mathrm{~h}$ with only a 0.01 loss in the accuracy with prephased haplotypes for imputation of the test samples using IMPUTE2 (Howie et al., 2012). Nevertheless, FImpute seems a good choice for imputation from $54 \mathrm{~K}$ to $\mathrm{HD}$, considering both imputation accuracy and computational demand, as both the reference and test populations could be simultaneously updated in a dairy cattle population.

When imputing from $3 \mathrm{~K}$ to $54 \mathrm{~K}$, findhap, AlphaImpute, and FImpute obtained lower accuracies than IMPUTE2 and Beagle, although these 3 methods also used the available pedigree information. The possible reasons could be as follows: (1) they did not use the population information for imputation as efficiently as IMPUTE2 and Beagle did, (2) the family information was weaker in this study because no genotyped dams were used (in fact, only $0.041 \%$ missing genotypes were imputed in the family step when using FImpute for imputation from $3 \mathrm{~K}$ to $54 \mathrm{~K}$ ), or (3) the population was an admixture population that combined Swedish Red and Finish Red. On the other hand, these methods have the advantage that they can impute genotypes for nongenotyped individuals that are strongly related to genotyped individuals. This can be used for imputing genotypes of old but important bulls where DNA material is not available. An important advantage of FImpute and findhap is also their low computational demand. This is very attractive when large numbers of females and bull calves are genotyped at lower density.

\section{Influence of Relatedness Between Test and Reference Animals on Imputation Accuracy}

The relationship between test animals and reference animals affected the accuracy of imputation for all methods. Similar results have been reported in previous studies (Druet et al., 2010; Hickey et al., 2012a; Mulder et al., 2012). The relatedness reflects the probability of chromosome segments shared between reference and test animals. When the relatedness is stronger, the individuals have a greater probability of sharing more and longer haplotypes. As a result, the imputation of missing genotypes is more accurate. It was found that the methods that used pedigree information were more sensitive to the relationship between test and reference individuals than IMPUTE2 and Beagle. This indicates that different methods use family information and population information in different ways, and the superiority of different methods may depend on population structure and on the relatedness between the animals genotyped with different chips. In most countries, the sire and MGS of all new candidates are already genotyped. For the animals with both their sire and MGS in the reference population, the difference between imputation accuracies using different methods was relatively small, but IMPUTE2, BEAGLE, and FImpute still performed better than findhap and AlphaImpute. Furthermore, many countries have started to genotype some females. Including genotyped dams in the reference is expected to further improve the imputation accuracy.

\section{Imputation Accuracy Versus MAF}

Allele CR and correlation coefficients were used to measure the accuracy of imputation. The 2 measures rank methods in the same order in relation to imputation accuracy across all markers. However, in the comparison of imputation accuracies for markers with different MAF, the 2 measures showed opposite patterns. When MAF was low, the CR was high, whereas the correlation coefficient was low. The conflicting patterns are determined by the features of the 2 measures. Allele CR does not consider correct filling by chance, which is favorable for markers with low MAF. For example, given a marker with MAF of 0.01 , the marker can get a CR of $99 \%$ by chance (e.g., just by simply replacing the missing allele types with the major allele). On the contrary, the correlation coefficient is greatly influenced by extreme values. A few imputation errors for a marker with low MAF can greatly reduce the correlation coefficient for this marker. For example, given a $\mathrm{CR}$ of $95 \%$ for each genotype (AA, $\mathrm{AB}$, and $\mathrm{BB}$ ), the correlation coefficient is 0.802 for a marker with MAF of 0.05 , but 0.960 for a marker with MAF of 0.40 . In other words, the correlation is better at capturing the difference between imputation accuracy and correct filling by chance, which is important for estimation of imputation accuracy for markers with low MAF. It appears that compared with the allele $\mathrm{CR}$, the correlation coefficient better captures the differences of different methods and is also more appropriate when comparing the efficiency of imputing markers with low MAF. In this study, it was found that different imputation approaches had different sensitivity to MAF. Method IMPUTE2 showed the smallest changes with the change 
in MAF, followed by Beagle. This indicates that these 2 methods are more robust and better for imputation of missing genotypes at marker loci with low MAF.

It was found that the allele CR was lower for imputation of rare alleles than for common alleles. For a marker with low MAF, although the CR in this locus could be high, the CR for the minor allele could be low. This would greatly reduce the power to detect QTL as the rare alleles may play an important role in complex traits (Manolio et al., 2009). Therefore, for GWAS, the efficiency of imputing markers with low MAF is important.

\section{CONCLUSIONS}

Methods IMPUTE2 and Beagle are accurate and robust methods for imputing missing markers for individuals genotyped with various SNP chips in an admixed population without genotyped dams. The 2 methods were superior to findhap, FImpute, and AlphaImpute for imputation from $3 \mathrm{~K}$ to $54 \mathrm{~K}$, because they (1) give higher overall accuracy, (2) use population LD information efficiently to impute markers for individuals without close relatives in the reference population. However, FImpute could be a good choice for imputation from $54 \mathrm{~K}$ to HD, based on both imputation accuracy and computational demand. The $54 \mathrm{~K}$ panel can be accurately imputed to the HD panel. On the other hand, the $3 \mathrm{~K}$ panel is not dense enough to be imputed to the $54 \mathrm{~K}$ panel with satisfactory accuracy in the Swedish and Finnish Red population. A denser chip (e.g., BovineLD; 7K) should be considered. Relatedness between test animals and reference animals has a substantial influence on imputation accuracy, but is more profound when using findhap, AlphaImpute, and FImpute, as these rely on pedigree information. Alleles with low frequency were more difficult to impute, but this is hard to see when using the allele CR as a measure of accuracy. Therefore, the correlation coefficient is a better measure of imputation efficiency, as it better captures the difference between imputation accuracy and correct filling by chance than the $\mathrm{CR}$.

\section{ACKNOWLEDGMENTS}

We acknowledge the Danish Cattle Federation (Aarhus, Denmark), Faba Co-op (Helsinki, Finland), Swedish Dairy Association (Stockholm, Sweden), and Nordic Cattle Genetic Evaluation (Aarhus, Denmark) for providing data. This work was performed in the project "Genomic Selection-From function to efficient utilization in cattle breeding (grant no. 3405-10-0137)," funded under Green Development and Demonstration Programme by the Danish Directorate for Food, Fish- eries and Agri Business (Copenhagen, Denmark), the Milk Levy Fund (Aarhus, Denmark), VikingGenetics (Randers, Denmark), Nordic Cattle Genetic Evaluation (Aarhus, Denmark), and Aarhus University (Aarhus, Denmark). We thank John Hickey (University of New England, Australia) and Mehdi Sargolzaei (University of Guelph, Ontario, Canada) for their help in implementing the imputation software and valuable discussion.

\section{REFERENCES}

Boichard, D., H. Chung, R. Dassonneville, X. David, A. Eggen, S. Fritz, K. J. Gietzen, B. J. Hayes, C. T. Lawley, T. S. Sonstegard, C. P. Van Tassell, P. M. VanRaden, K. A. Viaud-Martinez, and G. R. Wiggans. 2012. Design of a bovine low-density SNP array optimized for imputation. PLoS ONE 7:e34130.

Brøndum, R. F., P. Ma, M. S. Lund, and G. Su. 2012. Short communication: Genotype imputation within and across Nordic cattle breeds. J. Dairy Sci. 95:6795-6800.

Brøndum, R. F., E. Rius-Vilarrasa, I. Strandén, G. Su, B. Guldbrandtsen, W. F. Fikse, and M. S. Lund. 2011. Reliabilities of genomic prediction using combined reference data of the Nordic Red dairy cattle populations. J. Dairy Sci. 94:4700-4707.

Browning, B. L., and S. R. Browning. 2009. A unified approach to genotype imputation and haplotype-phase inference for large data sets of trios and unrelated individuals. Am. J. Hum. Genet. $84: 210-223$.

Browning, S. R., and B. L. Browning. 2007. Rapid and accurate haplotype phasing and missing-data inference for whole-genome association studies by use of localized haplotype clustering. Am. J. Hum. Genet. 81:1084-1097.

Dassonneville, R., R. F. Brøndum, T. Druet, S. Fritz, F. Guillaume, B. Guldbrandtsen, M. S. Lund, V. Ducrocq, and G. Su. 2011. Effect of imputing markers from a low-density chip on the reliability of genomic breeding values in Holstein populations. J. Dairy Sci. 94:3679-3686.

Dassonneville, R., S. Fritz, V. Ducrocq, and D. Boichard. 2012. Short communication: Imputation performances of 3 low-density marker panels in beef and dairy cattle. J. Dairy Sci. 95:4136-4140.

Druet, T., and M. Georges. 2010. A hidden Markov model combining linkage and linkage disequilibrium information for haplotype reconstruction and quantitative trait locus fine mapping. Genetics 184:789-798.

Druet, T., C. Schrooten, and A. P. de Roos. 2010. Imputation of genotypes from different single nucleotide polymorphism panels in dairy cattle. J. Dairy Sci. 93:5443-5454.

Habier, D., R. L. Fernando, and J. C. M. Dekkers. 2009. Genomic selection using low-density marker panels. Genetics 182:343-353.

Hayes, B. J., P. J. Bowman, A. J. Chamberlain, and M. E. Goddard. 2009. Invited review: Genomic selection in dairy cattle: Progress and challenges. J. Dairy Sci. 92:433-443.

Hickey, J. M., J. Crossa, R. Babu, and G. de los Campos. 2012a. Factors affecting the accuracy of genotype imputation in populations from several maize breeding programs. Crop Sci. 52:654-663.

Hickey, J. M., B. P. Kinghorn, B. Tier, J. H. van der Werf, and M. A. Cleveland. 2012b. A phasing and imputation method for pedigreed populations that results in a single-stage genomic evaluation. Genet. Sel. Evol. 44:9.

Howie, B., C. Fuchsberger, M. Stephens, J. Marchini, and G. R. Abecasis. 2012. Fast and accurate genotype imputation in genome-wide association studies through pre-phasing. Nat. Genet. 44:955-959.

Howie, B., J. Marchini, and M. Stephens. 2011. Genotype imputation with thousands of genomes. G3: Genes, Genomes, Genetics $1: 457-470$

Howie, B. N., P. Donnelly, and J. Marchini. 2009. A flexible and accurate genotype imputation method for the next generation of genome-wide association studies. PLoS Genet. 5:e1000529. 
Johnston, J., G. Kistemaker, and P. G. Sullivan. 2011. Comparison of different imputation methods. Interbull Bull. 44:25-33.

Lund, M. S., A. P. W. de Roos, A. G. de Vries, T. Druet, V. Ducrocq, S. Fritz, F. Guillaume, B. Guldbrandtsen, Z. T. Liu, R. Reents, C. Schrooten, F. Seefried, and G. S. Su. 2011. A common reference population from four European Holstein populations increases reliability of genomic predictions. Genet. Sel. Evol. 43:43.

MacLeod, I. M., B. J. Hayes, K. W. Savin, A. J. Chamberlain, H. C. McPartlan, and M. E. Goddard. 2010. Power of a genome scan to detect and locate quantitative trait loci in cattle using dense single nucleotide polymorphisms. J. Anim. Breed. Genet. 127:133-142.

Manolio, T. A., F. S. Collins, N. J. Cox, D. B. Goldstein, L. A. Hindorff, D. J. Hunter, M. I. McCarthy, E. M. Ramos, L. R. Cardon, A. Chakravarti, J. H. Cho, A. E. Guttmacher, A. Kong, L. Kruglyak, E. Mardis, C. N. Rotimi, M. Slatkin, D. Valle, A. S. Whittemore, M. Boehnke, A. G. Clark, E. E. Eichler, G. Gibson, J. L. Haines, T. F. C. Mackay, S. A. McCarroll, and P. M. Visscher. 2009. Finding the missing heritability of complex diseases. Nature 461:747-753.

Matukumalli, L. K., C. T. Lawley, R. D. Schnabel, J. F. Taylor, M. F. Allan, M. P. Heaton, J. O'Connell, S. S. Moore, T. P. L. Smith, T. S. Sonstegard, and C. P. Van Tassell. 2009. Development and characterization of a high density SNP genotyping assay for cattle. PLoS ONE 4:e5350.

Meuwissen, T. H. E. 2009. Accuracy of breeding values of 'unrelated' individuals predicted by dense SNP genotyping. Genet. Sel. Evol. 41:35.

Meuwissen, T. H. E., B. J. Hayes, and M. E. Goddard. 2001. Prediction of total genetic value using genome-wide dense marker maps. Genetics 157:1819-1829.

Mulder, H. A., M. P. L. Calus, T. Druet, and C. Schrooten. 2012. Imputation of genotypes with low-density chips and its effect on reliability of direct genomic values in Dutch Holstein cattle. J. Dairy Sci. 95:876-889.

Sargolzaei, M., J. P. Chesnais, and F. S. Schenkel. 2011. FImpute An efficient imputation algorithm for dairy cattle populations. J. Dairy Sci. 94(E-Suppl. 1):421. (Abstr.)

Segelke, D., J. Chen, Z. Liu, F. Reinhardt, G. Thaller, and R. Reents. 2012. Reliability of genomic prediction for German Holsteins using imputed genotypes from low-density chips. J. Dairy Sci. 95:5403-5411.

Su, G., R. F. Brøndum, P. Ma, B. Guldbrandtsen, G. P. Aamand, and M. S. Lund. 2012. Comparison of genomic predictions using medium-density $(\sim 54,000)$ and high-density $(\sim 777,000)$ single nucleotide polymorphism marker panels in Nordic Holstein and Red Dairy Cattle populations. J. Dairy Sci. 95:4657-4665.

$\mathrm{Su}$, G., B. Guldbrandtsen, V. R. Gregersen, and M. S. Lund. 2010 Preliminary investigation on reliability of genomic estimated breeding values in the Danish Holstein population. J. Dairy Sci. 93:1175-1183.

Sun, C., X. Wu, E. A. Weigel, U. J. Rosa, S. Bauck, B. W. Woodward, R. D. Schnabel, E. F. Taylor, and D. Gianola. 2012. An ensemblebased approach to imputation of moderate-density genotypes for genomic selection with application to Angus cattle. Genet. Res. (Camb.) 94:133-150.

VanRaden, P. M., J. R. O'Connell, G. R. Wiggans, and K. A. Weigel. 2011. Genomic evaluations with many more genotypes. Genet. Sel. Evol. 43:10.

VanRaden, P. M., and P. G. Sullivan. 2010. International genomic evaluation methods for dairy cattle. Genet. Sel. Evol. 42:7.

Wiggans, G. R., T. A. Cooper, P. M. VanRaden, K. M. Olson, and M. E. Tooker. 2012. Use of the Illumina Bovine3K BeadChip in dairy genomic evaluation. J. Dairy Sci. 95:1552-1558. 